\title{
Theory and Experiment of Cooperative Control at Multi-Intersections in Intelligent Connected Vehicle Environment: Review and Perspectives
}

\author{
Linan Zhang ${ }^{1,2}$, Yizhe Wang ${ }^{1,2, *(1)}$ and Huaizhong $\mathrm{Zhu}^{3}$ \\ 1 Key Laboratory of Road and Traffic Engineering, Ministry of Education, Tongji University, \\ Shanghai 201804, China; 1410080@tongji.edu.cn \\ 2 Intelligent Transportation System Research Center, Tongji University, Shanghai 201800, China \\ 3 School of Artificial Intelligence, Tianhua College, Shanghai Normal University, Shanghai 201815, China; \\ 1310746@tongji.edu.cn \\ * Correspondence: 16wangyizhe@tongji.edu.cn; Tel.: +86-21-188-173-69234
}

check for updates

Citation: Zhang, L.; Wang, Y.; Zhu, H. Theory and Experiment of Cooperative Control at Multi-Intersections in Intelligent Connected Vehicle Environment: Review and Perspectives.

Sustainability 2022, 14, 1542.

https://doi.org/10.3390/su14031542

Academic Editors: Péter Gáspár and Tamás Bécsi

Received: 6 January 2022

Accepted: 25 January 2022

Published: 28 January 2022

Publisher's Note: MDPI stays neutral with regard to jurisdictional claims in published maps and institutional affiliations.

Copyright: () 2022 by the authors Licensee MDPI, Basel, Switzerland. This article is an open access article distributed under the terms and conditions of the Creative Commons Attribution (CC BY) license (https:// creativecommons.org/licenses/by/ $4.0 /)$.

\begin{abstract}
A heterogeneous traffic flow consists of regular vehicles, and intelligent connected vehicles having interactive functions is updating the composition of the current urban-road network traffic flow. It has been a growing trend and will continue to be so. Because of the urgent demand, the research focused on three main parts of cooperative control methods under intelligent connected vehicles environment, typical traffic control application scenarios and experimental validation in intelligent connected vehicles conditions, and intersection-oriented hybrid traffic control mechanism for urban road. For heterogeneous interrupted traffic flow of intelligent connected vehicles, to analyze the characteristics and information extraction method of heterogeneous traffic flow of intelligent connected vehicles under different conditions, the research examined driving modes of regular vehicles and intelligent connected vehicles, including car following and lane changing. This study summarized control modes of traffic-signal control, active control of intelligent connected vehicles, and indirect control of regular vehicles through intelligent vehicles to study the active control mechanism and multi-intersection coordinated control strategy for intelligent connected vehicle heterogeneous traffic flow. With the combination of coordinated control theory, this work overviewed integrated experiment of information interaction and coordinated control under intelligent-connectedvehicle heterogeneous traffic-flow environments.
\end{abstract}

Keywords: intelligent transportation; intelligent connected vehicles; cooperative traffic control; connected-level multi-intersections; typical application scenarios

\section{Introduction}

With the further development and promoting application of intelligent-connected vehicles and cooperative vehicle infrastructure technologies, an integrated human-vehicleinfrastructure transportation system will come true. In the condition of cooperative perception, fusion, and interaction of all space-time traffic information, it is urgent to conduct research on the theories, methods, and key technologies for cooperative control of multiintersections under the environment of intelligent-connected vehicle traffic flow. The goal of the traffic control mechanism in the intelligent-connected vehicle environment is to create an intelligent transportation system, using technology such as intelligent-connected vehicles and achieving the organic combination between "smart vehicle" and "intelligent infrastructure" to ensure the proper functioning of the urban transportation system. In summary, the study has a clear direction, frontier, and trend, which is of strategic significance and practical value to further promote the development and application of the next-generation traffic-network control system to seize the high ground in this field. 
The concept of the vehicle-road cooperation system came into being at the beginning of the 21st century. Afterwards, the driving-safety technology of the vehicle-road cooperation system was recognized as a new generation of traffic-safety generation following seat belts and airbags. Demonstration systems based on this concept include Mcity in the United States, ETPC in the Netherlands, AstaZero in Sweden, and Jtown in Japan.

With the continuous development of vehicle-road collaboration technology, intelligent connected vehicle technology has been further promoted and applied. A new type of heterogeneous traffic flow composed of regular vehicles and intelligent connected vehicles has appeared and will continue to exist, and the traffic-flow structure of the urban road network will also change accordingly. In the mixed-traffic environment, driving behaviors and traffic-flow-control requirements will change. Therefore, the research of the multimode traffic-flow-control theory was imperative. Several forward-looking studies have theoretically explored the impact of connected vehicles and automated vehicles on traffic flow, but the research on multimode traffic-flow control and its experiment are rarely conducted.

\section{Traffic Parameters Extraction and State Analysis under Intelligent-Connected Environment}

In recent years, scholars in related fields have studied traffic parameter extraction methods based on intelligent connected vehicles to solve the defects of traditional datacollection methods, and some new methods continue to emerge. It mainly focused on extracting traffic parameters that cannot be directly obtained by traditional detectors, such as traffic density, average speed, and travel time, and has made major breakthroughs in timeliness and accuracy of data.

\subsection{Traffic Parameters Extraction under Intelligent-Connected Environment}

Sooksan [1] et al. proposed a traffic-density estimation method based on the vehicle number near the probe vehicle under the vicinity of a vehicle in a vehicular Ad hoc network (VANET) environment. The core of the method was to estimate the global density based on the local density; that is, we calculated the density of a small area based on the number of vehicles of the network and then estimated the traffic density of the whole road. The study was later extended to a clustering approach [2] whereby the global density was estimated by collecting information on cluster members through cluster heads. The disadvantage of the above model only works if the head spacing obeyed exponential distribution, which was less applicable, and the model only considered the vehicles behind the detection vehicle. The detection vehicle can receive data information from all directions within the effective communication range.

Fogue, Barrachina, and Sanguesa et al. proposed, respectively, a real-time trafficdensity estimation method based on VANET, using V2V [3], V2I [4,5], and V2X [6] techniques. The model considered the number of beacons received by each vehicle or RSU and the topology of the urban road network (number of roads and number of intersections) and other characteristics to obtain a traffic density calculation model by using linear regression analysis. The simulation experiments verified that the regression model can estimate the traffic density of any given city well. In addition, Sanguesa [7] optimized and improved the traffic-density estimation model based on the above study by considering the effect of the total length of the road network. The simulation results showed that the improved model can better describe the traffic density of road network. However, these models above study on the macro level, i.e., the average traffic density of the whole road network, and cannot obtain the density of a specific road section. It was not very valuable for practical applications. Because of the complex and variable nature of urban road traffic, different road sections often have different traffic densities, and traffic control and management work were more concerned with the real-time traffic density of a specific road section.

Arbabi [8] used the ICV technique to extract some common traffic data, such as time mean speed, space mean speed, traffic flow, and travel time. Simulation results showed that the method can obtain accurate traffic parameters at $100 \%$ loading rate. The study was later further refined [9]. The authors built a Dynamic Traffic Monitoring System 
(DTM System) in an ICV environment, using an RSU to collect and send road-status information to vehicles loaded with OBU devices to extract travel time and average speed in a free-flow environment. The simulation results showed that high-quality travel time and mean speed can be obtained even at a low loading rate. The disadvantage was that the authors investigated the accuracy of traffic-parameter extraction only in the free-flow traffic environment and did not consider the applicability of the model in other traffic environments, such as congestion and others. Christian [10] used V2I communication to periodically send messages containing the current location, speed, ID, and timestamp of the vehicle through the OBU, and the RSU received and processed these messages.

\subsection{Traffic State Analysis under Intelligent-Connected Environment}

$\mathrm{Li}$ [11] proposed the mean speed of road section, mean speed of intelligent connected vehicles, and machine-learning aggregated values as variables for road-section meanspeed estimation for mixed scenarios of connected vehicles and non-connected vehicles, respectively. Moreover, he found that the accuracy of each estimation increases with the increase of the occupancy of intelligent connected vehicles in road section according to the mean-square-error distribution. Chen [12] collected three traffic parameters, namely, vehicle mean speed, one-way traffic density, and one-way travel time, and then established a traffic state evaluation model and a single-parameter traffic-state evaluation index model based on fuzzy theory and verified the feasibility of the model through simulation. The disadvantage was that, because of the separated simulation, the data cannot reproduce the vehicle-road cooperative environment, and no comparative study was performed on the accuracy of the models. Liu [13] established a Block model of microscopic road network. Based on the information of vehicles positioned in the Block, traffic parameters, such as density, speed, and flow, were extracted. The disadvantage was that the study only reproduces the single vehicle motion trajectory and the road network traffic state evolution process through separated simulation experiments, without directly verifying the accuracy of the traffic parameter calculation model; in addition, the whole model assumes of positioning accuracy up to $1 \mathrm{~cm}$, which is difficult to meet in practice. In his master thesis, Nie [14] analyzed the relationship between traffic flow and information flow in ICV environment from both qualitative and quantitative perspectives. On the one hand, the communication simulation software named NS-3 and the traffic simulation software called VanetMobiSim were used to study the influence of traffic flow on information flow under three typical traffic scenarios, respectively; on the other hand, the simulation software named EstiNet was used to study the influence of information flow on traffic flow. By studying the extraction method of average travel time, a regression model was established to obtain the mutual-influence relationship between average travel time, detour rate, and three other factors-obedience rate, communication distance, and equipment loading rate. The simulation results showed that the detour rate increases with the increase of the above three factors, and the average travel time decreases with the increase of them. There were two main shortcomings in this study: first, the simulation was loosely coupled, and the trace data obtained from the traffic simulation software were imported into the NS-3 communication simulation software, which did not realize the simultaneous simulation of traffic and communication, and the accuracy of the simulation was not high; second, the actual road traffic conditions were not considered, so the regression model of average travel time lacks practical significance, and the accuracy of the model was not verified. The applicability of the model was not known.

In the literature, Li [15] proposed a real-time travel time estimation (TTE) method between intersections based on ICV technology. The model validation was calculated by selecting historical data and compared with real cab data. The average accuracy of the model was obtained as $86.4 \%$. However, the model only considered historical data sources and did not consider the real-time nature of the data; if real-time data sources are added to the model, its accuracy can be further improved. Zhou [16] studied the traffic information collection and processing method on the Internet of Vehicles environment. He used an 
improved support vector regression method and an improved artificial neural network method to predict the travel time on the Internet of Vehicles environment and evaluated the prediction accuracy under different penetration rates and episodic blockages. However, the simulation of OBU vehicles in the paper was only designated as a special vehicle type, which was not truly connected vehicle; secondly, the simulation data were collected by using data from the traditional traffic environment, which cannot fully represent the characteristics of the Internet of Vehicles environment.

In summary, although some achievements have been made by scholars in related fields in the acquisition of traffic parameters in the intelligent connected vehicle environment, there were still deficiencies of different degrees, mainly in the following aspects.

(1) In terms of data sources: Some studies use real data from traditional traffic environment or static data obtained from separated simulation environment, both of which cannot reflect the information interaction environment of intelligent connected vehicles. In addition, some studies only used historical data, and the data sources lacked real-time data.

(2) In terms of location accuracy: To obtain high-quality traffic parameters in the intelligent connected vehicle environment, some of the studies assume of fine-grained location information and lack of research on vehicle-location accuracy.

(3) In terms of simulation method: Affected by the difficulty of simulation, most of the studies adopt the separated simulation method, and the trace data obtained from the traffic-simulation software were imported into the communication simulation software, which did not realize the synchronous simulation of communication and traffic, and the simulation accuracy was not high.

(4) In terms of model validation: Some studies did not verify the accuracy of the model, but only explored the feasibility of the model.

(5) In terms of applicability: Most of the relevant studies only considered the acquisition of traffic parameters in the free-flow environment and did not consider the impact on the model under non-constant traffic conditions, such as congestion [9].

Therefore, to address the shortcomings of existing related research, specific research work will be carried out in the future on fine-grained location information, estimation models of traffic parameters under normal and non-normal traffic conditions, and model accuracy validation in the intelligent connected vehicle environment, using both real-time and historical data in a tightly coupled simulation approach.

\section{Analysis of Traffic Flow Operation Characteristics in Intelligent Connected Vehicles Environment}

\subsection{Research Review of Car-Following Model}

The study of the car-following theory started in the 1950s. In 1950, Reushel and Pipes [17] studied the car-following process, marking the beginning of the research on the analytical methods of car-following theory. The GM series models proposed by Gazis, Herman, Rothory, and others [18] at the General Dynamics Laboratory, around 1960, became the most important work in the early research of the car-following theory.

With the refinement of data collection, car-following models based on many data models have emerged in recent years, including cloud-theory-based car-following models, fuzzy logic-based car-following models, and neural-network-based car-following models $[19,20]$.

Goodall et al. [21] proposed a method based on the Wiedeman car-following model to estimate connected vehicles on the road. Based on Goodall's research, Feng [22] retained the Widedman car-following model's method of estimating connected vehicles. The estimated position and speed data of connected vehicles were used in real-time signal control, resulting in a significant reduction in the total delay of the vehicle.

Siyuan Gong and Lili Du [23] studied the cooperative fleet control methods under the environment of mixed traffic of intelligent connected vehicles and non-intelligent connected vehicles. The Newell model was used to modify the car-following model of heterogeneous traffic flow and proposed a curve-fitting algorithm that can predict delays of non-intelligent vehicles based on real-time trajectory data. 


\subsection{Research Review of Lane-Changing Model}

The lane-changing model was one of the two most basic dynamic models in microtraffic simulation. The lane-changing process was a process that requires comprehensive judgment and operation, and it was more difficult to determine the vehicle acceleration during the lane-changing process. There were two main types of lane-changing models: discretionary lane-changing models and imperative lane-changing models. Currently, the most widely used lane-changing models include the Gipps model [24], MITSIM model [25], CORSIM model [26], SITRAS model [27], etc. In addition, the cellular automata model based on microscopic individual rules was used in multi-lane traffic flow. The results showed that, compared with the lane-changing model, without continuous planning, this model improved the authenticity of the lane-changing behavior of a single vehicle.

Sun et al. [28] established a new lane-changing model that included competition and cooperation behavior and quantified the interaction of different vehicles in the lane-change process. By implementing and validating the model in the CORSIM micro-simulator package and comparing it with the original lane-changing model in CORSIM, the results showed that the model can better simulate the lane-changing process under different congestion levels. Talebpour et al. [29] proposed a lane-changing model based on a gametheoretic approach considering that vehicles can receive information from surrounding vehicles in Internet-of-Vehicles environment. The simulation results showed that the new lane-changing model better simulates the real lane-change situation due to the inclusion of multi-vehicle interaction effects.

The cellular automaton model was an effective tool to study the dynamic characteristics of traffic flow in urban-road networks and can reproduce many important characteristics of road traffic (e.g., traffic volume, average speed, density, etc.). Nowadays, the theory of cellular automata has been widely applied in transportation research. The most famous one was the one-dimensional cellular automata NaSch single-lane model proposed by German scholars Nagel and Schreckenberg [30]. The basic principle of this model was to simulate a single lane as a one-dimensional grid where each grid represents a cell, and the non-empty cell indicates that the road was occupied by vehicles. The single lane requires that vehicles can only follow the vehicle ahead and do not overtake. To better reflect the real road traffic flow state, Biham, Middleton, and Levine [31] proposed a two-dimensional cellular automaton BML model which identified the basis for studying traffic flow, using the cellular automaton model.

Kohei Arai et al. [32] used cellular automata to study the impact of lane-changing actions on the driver's perception. The simulation results showed that the model can better capture the important features of lane-changing actions, and it also reflected the impact of lane-changing on road congestion. Wang [33] considered the influence of the driver's psychology and behavior, introduced two quantitative indicators of intention intensity and risk factor, and proposed a multi-lane lane changing model that couples the driver's intention and driving tendency. Deng et al. [34] introduced a one-dimensional cellular automata model for improved comfortable driving and formed a multi-lane cellular automata model based on the decision-making mechanism of lane changing.

Zhao et al. [35] summarized the effect between multiple vehicles as the probability of drivers' intention to change lanes. Li et al. [36] proposed a competitive lane-changing model based on the theory of cellular automata, in which the lane-changing vehicles and the surrounding vehicles compete to exercise the priority during the lane-changing implementation. With the rapid development of $5 \mathrm{G}$ communication technology, cooperative motion control was adopted between vehicles to avoid collisions and made the traffic flow more smooth and safer.

At present, there was no unified concept about the cooperative lane-changing strategy. Hidas [37] and Kelsch [38] from the University of New South Wales, Australia; Yunlong Tan from Jilin University; and Matthias [39] from the German Aerospace Center have all given the concept of a cooperative loop. 
Traffic-state analysis was the basis of traffic-system theory and traffic control and management. Since the previous traffic-information acquisition was mainly based on fixed [40] and mobile low-frequency sampling methods [41] with spatial-temporal discontinuity and incompleteness, much research was mostly devoted to the fields of optimizing traffic-information quality [42], restoring the real traffic state [43] and its prediction [44]. The vehicle-road connected environment made it possible to obtain more precise and comprehensive traffic data in a wider spatial and temporal dimension [45]. Therefore, scholars have studied traffic-flow detection [46], travel-time estimation [47], intersectionqueue-length estimation [48], traffic-state identification, and intersection vehicle-behavior recognition in the new information environment. The research of Goodall et al. [49] showed that the algorithm was able to predict the location of $30 \%$ of non-connected vehicles in the same lane with $9 \mathrm{~m}$ accuracy in the presence of only $10 \%$ of connected vehicles. ArgoteCabañero et al. [50] investigated the minimum penetration rate of a connected vehicle that can accurately estimate the measures of effectiveness (MOEs) of signal-control arterial roads under under-saturation and over-saturation conditions, such as average speed, number of stops, acceleration, delay, etc. Tests in a simulated section of the San Pablo Avenue main road in Berkeley, California, showed that the efficiency index under most traffic conditions requires a minimum penetration rate of interconnected vehicles of less than $1 \%$. Based on the traffic data of multiple road sections in a road network area, Zhang Yi's team at Tsinghua University clustered the traffic data into related clusters, using a self-organizing maps (SOMs) model to identify and analyze traffic-data patterns in the spatial-temporal domain and analyze the spatial and temporal-evolution patterns of traffic states [51].

Ran's team [52] of Southeast University combined vehicle-road cooperation and Internet technology to divide the traffic model into four development stages and discussed the impact of ITS technology on the traffic-flow model and traffic-control model in the future development stages. Zhao's team [53] of Chang'an University proposed a hybrid traffic-flow prediction model containing nonparametric regression and BP neural network for the characteristics of periodic and random changes of short-time traffic flow and conducted experiments on the traffic flow data of a road section in Xi'an city. Wang's team [54] of Beijing University of Aeronautics and Astronautics proposed an automatic on-ramp control algorithm under the condition of fully connected vehicles and conducted simulation analysis for single-vehicle merging and two-vehicle merging, respectively. Yao's team [55] from Tsinghua University addressed the commercial GPS positioning delay problem and calculated the GPS positioning delay by comparing the correlation degree between velocity difference time series and acceleration time series to obtain real-time vehicle-location information. These studies showed the evolution law research based on multi-modal vehicle traffic flow data oriented to traffic-signal control.

In the past, the car-following and lane-changing theory used the method of fluid dynamics to study the theory that the motion state of the rear vehicle changes due to the change of the front vehicle state when the vehicle is in procession on the road, and it is unable to change lanes and overtake. The mathematical model is used to express the various states that occur in the process of car-following. Drivers rely on their perception and judgment to respond to a series of continuous state changes in front of the car. The carfollowing theory tries to understand the characteristics of the one-lane traffic flow by observing the vehicles following one by one. The study of this characteristic can be used to describe the stability of traffic flow, acceleration disturbance, and propagation of disturbance. In the information interactive environment, the car-following model can test the management technology and communication technology to minimize the rearend collision.

\section{Cooperative Control Methods under Intelligent Connected Vehicles Environment}

With the development of intelligent connected vehicle technology, on the one hand, real-time and reliable vehicle-vehicle/vehicle-road communication has become possible; on the other hand, traffic congestion and other problems have put forward higher require- 
ments for traffic control. How to improve the existing traffic-control system based on section detection data, how to introduce vehicle-road cooperation technology, and how to use its two-way data communication to improve the benefits of traffic control have gradually become important issues to be researched and solved for the future development of urban traffic control system. Therefore, the research on active traffic control based on vehicle-road cooperation and intelligent connected vehicle technology must focus on the following questions: What changes does the incorporation of intelligent connected vehicles have on traditional traffic flow? How do we accurately extract the information needed by the traffic control system in the environment of intelligent connected vehicles? How do we create a cooperative control mechanism for multiple intersections in the environment of connected vehicles without interaction with the traffic control system? How doe we build intersection active control mechanism in the environment of intelligent connected vehicles with interaction function with traffic control system? How do we verify the cooperative control mechanism of multiple intersections in the environment of intelligent connected vehicles?

\subsection{Shortage of Existing Traffic-Control Methods}

The established control theories and methods are not suitable, including based on hypothetical conditions, unreliability of prediction, poor adaptability of models and algorithms, frequent phase switching, and fixed-phase sequence; control systems are, in essence, data-driven demand-responsive working mechanisms based on data, lacking induction mechanisms of the controlled party, etc. Five aspects, namely information collection, control strategy, information dissemination, control objectives, and control results, are compared with the differences between traffic signal control in the traditional environment and traffic control in the intelligent connected vehicle (information interaction) environment, respectively.

(1) Traffic signal control in traditional environments.

Information collection: section form detectors, which passively detect traffic disturbances.

Control strategy: model estimation prediction based on cross-sectional data, which cannot respond directly to traffic demand.

Information dissemination: through signal colors or variable message boards, unable to optimize information dissemination content for individuals.

Control objectives: the target parameters are mainly overall traffic flow delays or travel times, and the accuracy of the target parameter modeling is itself affected by information collection limitations.

Control results: In each signal cycle, there are still small changes in traffic demand, and the failure to respond accurately to them will result in wasted green-light time.

(2) Traffic control in an intelligent connected vehicle (information interaction) environment.

Information collection: continuous spatiotemporal trajectory data, capable of proactively sensing disturbances in the traffic system.

Control strategy: active vehicle guidance and optimized control based on real-time traffic-demand data.

Information dissemination: simultaneous dissemination through signal color and in-vehicle systems that can optimize information dissemination content for individuals.

Control objectives: In addition to traditional control objectives, green-light utilization, energy-consumption index, emission index, and fair distribution of traffic time and space resources can be considered.

Control results: In each signal cycle, adjustments can be made at a longer distance through speed guidance and signal priority to decrease green-light-time wastage.

\subsection{Changes to Traffic Control in the Intelligent Connected Vehicle Environment}

In the traditional traffic-signal control system, the controlled party (vehicle driver) can only passively adapt to the signal. With the emergence of intelligent connected vehicles, the control party and the controlled party can realize the real sense of information interaction, making the whole control system's formation of a full element of closed-loop 
feedback mechanism possible. Facing the three traffic control modes under the traffic-flow conditions of intelligent connected vehicles, namely signal control, intelligent connected vehicle guidance and induction, and indirect control of conventional vehicles through intelligent connected vehicles, etc., taking the minimum coordinated control networkmulti-intersection - as the control object, integrate the three control modes, and especially research to clarify the intersection multi-modal control mechanism under the intelligent connected vehicle traffic flow conditions. An iterative "Simple Model, Active learning" data-driven traffic-control method was developed to adapt to the cooperative mechanism of three control modes under the conditions of large-scale traffic data and intelligent connected traffic flow. The method can improve the global benefits of traffic control systems, exploit the traffic control potential, improve the efficiency of existing traffic control, and enhance the functionality and performance of the system.

To study the cooperative mechanism between control objectives and control strategies, we must look at the traffic states and control parameters at multiple intersections. According to the high interactivity and information sharing of intelligent connected vehicle traffic flow, the optimal cooperative control mechanism of traffic flow between multiple intersections is analyzed to achieve the basic goal of improving the stability and anti-interference capability of the traffic system and the overall operational efficiency. In particular, the key coordination parameters, "offset and multi-intersection traffic control parameters", are studied, and the optimal phase-coordination control strategy for multi-intersections is formed. Meanwhile, the user-optimal control methods, such as personalized green-light demand degree for intelligent connected vehicles, and the system-optimal control methods, such as minimizing the average delay of multi-intersection vehicles and maximizing the number of multi-intersection vehicles flowing out, are studied to establish a balanced cooperative relationship between user-optimal and system-optimal control methods.

Compared with traditional traffic control methods, the traffic control mechanism in the intelligent connected vehicle environment has the following characteristics: collect travel path information in real time; intelligent connected technology automatically calibrates model parameters; active control is achieved through prompt messages, assisted driving, and other measures; the content of traffic control will become more abundant due to the introduction of the intelligent connected environment, such as dynamic path guidance, lane change control, etc.

Vehicle-road, vehicle-vehicle (intelligent vehicle and non-intelligent vehicle), and vehicle groups may be able to achieve system-level cooperation through self-organization, under the condition that their cooperation subjects are highly intelligent. However, it is very difficult for quite a long period of time; therefore, a considerable degree of its organization and control is an important method and measure to achieve cooperation between vehicle-road, vehicle-vehicle, and vehicle groups. Through scientific and effective control can ensure the system to a manageable goal direction. Therefore, the cooperative traffic control in the environment of intelligent connected vehicles, on the one hand, is due to the significant application needs of intelligent connected vehicles and their technical environment tends to be practical, so that the previous traffic-control environment and concepts are constantly changing and there is a sprouting of new technologies and new systems; on the other hand, the new requirements of traffic control, such as responding to unexpected events, special traffic control requirements, and the cooperation between controlled object and its influencing elements, have led to the need for a new generation of traffic control technologies. From the perspective of traffic system functions and objectives, fluidity and efficiency are the two main targets of the traffic system; from the perspective of traffic system service targets, satisfying special traffic (intelligent connected vehicles) service requirements will inevitably affect the benefits of the whole system.

\subsection{Traffic Control Methods in the New Environment}

$\mathrm{Chu} \mathrm{T}$ [56] proposed a MARL algorithm for the deep reinforcement learning agent A2C in an adaptive traffic signal control environment. The multi-agent $\mathrm{A} 2 \mathrm{C}$ was compared with 
the agent $\mathrm{A} 2 \mathrm{C}$ and $\mathrm{Q}$-independent learning algorithms in a large comprehensive traffic grid and a large realistic traffic network in the city of Monaco under simulated peak hour traffic dynamics. The results showed that the optimality, robustness, and sample efficiency of the algorithm are better than other state-of-the-art decentralized MARL algorithms. Gong $Y$ [57] proposed a network-level decentralized adaptive signal control algorithm using a deep learning approach in a multi-agent reinforcement learning framework. The evaluation results show that the proposed deep-reinforcement-learning-based algorithm can reduce the travel time by $10.27 \%$ and the total delay by $46.46 \%$. Liang X [58] proposed a deep reinforcement learning model for traffic signal control in a connected vehicle environment. In the model, the whole intersection was divided into small grids to quantify complex traffic scenarios, and the temporal variation of traffic signals was modeled as a high-dimensional Markov decision process. The effectiveness of the model was verified by simulating the urban traffic network. Jin J [59] proposed an adaptive signal control system considering a multi-criteria strategy. A general multi-agents signal control operation modeling framework was presented in the paper, where the behavior of each cognitive subject was modeled by a constrained Markov decision process, and a reinforcement learning algorithm was applied to the solution of the MDP problem. The results showed that the signal controller can make intelligent timing decisions based on predefined strategy goals. The control approach can be further applied to decentralized large networks. Chuhui Yu, Yiheng Feng, and Herry Liu [60-67] proposed an active control theory and method for CAV-based traffic networks based on the trajectory data and trajectory control technology of $\mathrm{CAV}$, and they developed a series of research results. CAV-based equilibrium models over a traffic network were presented based on the vehicle trajectory optimization model with determined paths. Macroscopic routing and microscopic trajectory planning were combined in one framework. In the system optimal principle, a bi-level programming model was built to optimize vehicle routing and trajectories for minimum vehicle delay. In the user optimal principle, a model of routing and trajectory planning was proposed for individual vehicles, assuming that vehicles are selfish. Moreover, the CAV-based user equilibrium conditions are presented accordingly. Genetic algorithms and heuristic iterative algorithms are designed to solve the system and the user optimal models.

The traffic-control theory involved in this paper mainly refers to the theoretical system of traffic-signal control at road intersections. It has been studied since the 19th century. It uses signal lights to direct traffic on the road and to control the sequence of vehicles entering and leaving the intersection. So far, it has formed a relatively complete theoretical system. According to its control strategy, it can be divided into timing control strategy, induction control strategy, and adaptive control strategy. According to the control range, it can be divided into single-point signal control, line control, and area control. In the past, the final scheme of traffic signal control was characterized by four basic control parameters: phase sequence, cycle time, green signal ratio, and phase difference. The research of traffic signal control provides a theoretical basis for the safe and efficient allocation of traffic rights at intersections. Under the environment of vehicle-road network, coordination, and cooperation, the distribution and cooperation control of the right of way will tend to be holographic, interactive, and highly active, refined, precise, and intelligent. The related theories will evolve from time to time.

\section{Typical Traffic Control Application Scenarios and Experimental Validation in Intelligent Connected Vehicles Conditions}

Focusing on the objective demand of relieving urban traffic congestion and reducing energy consumption and emission, as well as facing the hybrid traffic flow of intelligent connected vehicles composed of conventional vehicles and intelligent connected vehicles and its predictable technical conditions and directions, this study takes the small cooperative control network - the multi-intersection-as the object, reveals the intermittent traffic-flow operation characteristics and evolution rules of multi-intersection traffic under the environment of hybrid traffic flow of intelligent connected vehicles, and analyzes the 
cooperative mechanism of traffic-signal control and traffic-flow operation state between multi-intersection. With the strong support of multidimensional and multi-granularity traffic data of the intelligent connected vehicle environment, the study incorporates three control modes, namely signal control, intelligent connected vehicle guidance, and indirect control of conventional vehicles through intelligent connected vehicles, and establishes the basic theory and practical method of active control and cooperation of urban multiintersection traffic with the aims of preventing traffic congestion, improving system stability, and enhancing anti-interference capability and overall operation quality. The preliminary exploration of the integrated experimental platform construction method of information interaction and cooperative control under the mixed-traffic-flow environment of intelligent connected vehicles further deepens the theoretical foundation of the next-generation traffic-control system from the network layer.

Therefore, the core of the study is to determine the traffic-control mechanism in three major different scenarios: signal control in the conventional vehicle environment, interactive guidance and control in the intelligent connected vehicle environment, and indirect control of non-intelligent vehicles through intelligent vehicles in the intelligent connected vehicle environment.

\subsection{Conventional Manually Driven Vehicle Environment}

Conventional manually driven vehicles cannot interact with traffic-control systems, must rely on traffic signals for control, and can only passively adapt to signal control schemes. At present, conventional human-driven vehicles occupy most of the world, and it will take decades for intelligent connected vehicles to completely replace conventional human-driven vehicles, during which the traffic signal control will continue to exist and play an important role.

Data collection: Coil bayonet aggregate type, homogenized data, direct access to traffic flow, instantaneous speed, etc.; and indirectly obtain queue length, travel time, etc.

Control method: Adjustment of cycle, offset, and split.

Optimization evaluation method: System-optimal oriented and open-loop control.

\subsection{Intelligent Connected Vehicle Environment}

The characteristics of intelligent connected vehicles are that they can realize the information interaction between vehicles and signal control systems with the help of vehicleroad internet technology; realize the mutual cooperation between intelligent connected vehicles as the controlled party and traffic control systems as the controlling party from the perspective of supply and demand; and realize conditionally locally the optimal user of the intelligent connected vehicles through active control mechanisms, such as intelligent speed guidance and intelligent connected vehicle signal priority, on the basis of the signal control scheme that has already achieved the optimal signal-control scheme of the system.

Data collection: Based on intelligent connected vehicles actively acquiring trajectorylevel data, and directly obtaining position, speed, acceleration, path, delay, traffic flow, queue length, travel time, etc.

Control method: The two-way information interaction of intelligent connected vehicle technology can actively guide the traffic flow and to break through the existing "passive adaptation" to achieve the purpose of "active control"; at that time, the concept of cycle and split still exists, but the magnitude of the absolute value change and the space for settlement are also greater.

Optimization evaluation method: This is implemented through the mutual game between the individual optimum and the system optimum to achieve the cooperation between the controlling party and the controlled party; meanwhile, the multi-intersections are adjusted cooperatively according to the relevance, thus realizing the closed-loop and feedback control. 


\subsection{Mixed Environment of Conventional Vehicles and Intelligent Connected Vehicles}

As the intelligent connected vehicles can obtain the global path information that conventional vehicles cannot obtain, the intelligent connected vehicles can take the optimal trajectory driving by adjusting the speed, acceleration, and other parameters under the system information prompt. With the intelligent connected vehicle indirectly controlling conventional vehicles, it can make the conventional vehicles that cannot receive the traffic control system information take the same optimal trajectory, driving with the intelligent connected vehicle and improve the utilization efficiency of the traffic system. At this time, the intelligent connected vehicles can be imaginatively called "the moving signal lights".

Data collection is based on intelligent connected vehicles actively acquiring trajectorylevel data, and directly obtaining position, speed, acceleration, path, delay, traffic flow, queue length, travel time, etc. We can achieve multi-source data fusion between parameterlevel data, feature-level data, and decision-level data.

Control method: Three control patterns, namely signal control, intelligent connected vehicle guidance, and indirect control of conventional vehicles through intelligent connected vehicles, work cooperatively with each other.

Optimization evaluation method: This includes a rough mutual game between the individual optimum and the system optimum to achieve the cooperation between the controlling party and the controlled party; meanwhile, the multi-intersections are adjusted cooperatively according to the relevance, thus realizing the closed-loop feedback control.

\subsection{Experimental Validation of Traffic Control System in an Intelligent Connected Vehicle Environment}

The Connected Vehicle [68] project in the United States conducted a feasibility test of vehicle-road connected data collection and application covering 3000 vehicles in Michigan. The CVIS project in Europe conducted system integration and testing experiments in six experimental areas of different scales in France and Belgium. Other research institutions have built an integrated vehicle-road connected experimental platform [69] for traffic control and realized data collection and signal priority control experiments in a vehicle-road connected environment, which provides the possibility of larger-scale typical application experiments for vehicle-road internet. At present, in a comprehensive view, Chinese domestic connected vehicles may have to deal with more than 700 scenarios, which can provide more than 100 major scenarios [70]. Besides focusing on testing in different real scenarios, the new-generation control system should also be tested in each real scenario through multiple trials to reduce the impact of system uncertainties, such as communication delays and changes in vehicle dynamics characteristics. In terms of simulation experiments, however, research on experimental methods of vehicle-road connected and co-simulation combining communication simulation and traffic simulation [71] were also being carried out [72-74]. It was necessary to verify whether a control system was truly safe and efficient in many scenarios with different traffic conditions, different road conditions, and different environmental conditions [75]. Therefore, different factors affecting traffic, including the dynamic characteristics of vehicles, such as speed and acceleration [76], weather [77], traffic flow state, and infiltration rate of connected vehicles [78], should be considered in simulation tests. The robustness, repeatability, and scenario complexity are requirements for next-generation control-system simulation tests [79]. Soomin Woo and Alexander Skabardonis developed the Flow-Aware platoon organization strategy, where the CAVs perform platoon organization conditionally on the local traffic state, i.e., a low flow and a high speed [80]. Under the proposed framework by Reference [81], the vehicular trajectories of CAVs can be controlled to maximize the utilization of the green time and reduce the startup lost time by using a highly efficient trajectory planning algorithm. The CAV platooning operation, in coordination with traffic signals, was also implemented such that CAVs can pass the intersection efficiently. Owing to the infinite possibilities offered by the connected environment for autonomous vehicles, many research and development institutions and companies that originally focused on non-connected autonomous vehicles, represented by Google, have gradually shifted to connected autonomous vehicles in recent years. Research 
on connected self-driving vehicles has gradually replaced non-connected vehicles as a research hotspot. At present, there were already universities and companies, including the University of Michigan, Ford, GM, Honda, Nissan, and Delphi, joining the Mcity project. It is fair to say that the research community and industry have now reached a consensus that connected autonomous vehicles are the future of self-driving vehicles.

\section{Intersection-Oriented Hybrid Traffic Control Mechanism for Urban Road}

\subsection{Trajectory Control}

Recent advances in wireless communications, artificial intelligence, and sensor systems have laid the groundwork for the development of interconnected and autonomous vehicles. Interconnected and self-driving vehicles show great potential in improving traffic safety and efficiency, and they have become important topics for policymakers, industry professionals, and researchers. Interconnected vehicle technology enables real-time information sharing between individual vehicles and infrastructure; automated vehicle technology enables vehicles to perform complex movements with the help of advanced sensors and control actuators. The combination of vehicle networking technology and automatic vehicle technology further realizes the optimization of multi-vehicle trajectory and provides a new method for traffic operation.

By designing the trajectories of the connected automated vehicles, the state-of-theart studies aim to improve traffic-flow performance factors, such as safety, comfort, and efficiency. In the case of an intersection, vehicles arriving at a red light may stop at a stop pole and speed up when the light turns green. This process will increase the travel time and fuel consumption of individual vehicles, while reducing the capacity of intersections. With proper trajectory planning, connected automated vehicles can slow down ahead of time to avoid stopping and queuing. Therefore, the path planning of connected automated vehicles at intersections has been widely studied, since it cannot only optimize the driving behavior of individual vehicles but also improve the traffic flow.

Aiming at the energy-saving and emission-reducing of an individual vehicle at the intersection, the planning method of connected automated vehicle trajectory is adopted in the development of an eco-driving system. Eco-driving systems usually provide individual vehicles with eco-speed curves based on the behavior prediction and traffic-signal timing of the vehicle in front of them. The current state of the front vehicles can be measured by V2X communication reception, or by onboard sensors and loop detectors from the connected automated vehicles. The longitudinal driving behavior model is used to predict the future driving trajectory. To reduce the acceleration, deceleration, and idle speed during the red signal, the optimal speed curve of each vehicle is usually generated in the model predictive control framework.

On the other hand, in full and partial CAV (Connected-Automated Vehicle) environments, studies about CAV-based traffic control at signalized intersections are also introduced in the CAV trajectory planning. It is used to ease congestion, reduce the risk of accidents, and reduce fuel consumption and emissions. In a complete CAV environment, by precisely controlling the track of each CAV at an intersection, conflicts between incompatible vehicles can be completely avoided without the need for traffic lights. In addition, if the CAV needs to wait for other vehicles at the intersection, it will plan the optimal trajectory strategy to reduce its speed in advance to avoid parking and queuing, which can eliminate the start-up loss and improve the driving experience. However, the complete CAV environment is not possible to achieve soon. Compared with the complete CAV environment, the trajectory planning in some CAV environments needs to consider the motion of HV, which cannot be controlled directly and accurately. In this mixed-traffic environment, some studies have designed a longitudinal velocity curve of CAVs based on the prediction of the HV's future trajectory. Elefteriadou et al. proposed an Intelligent Intersection Control System (IICS) for mixed traffic flow at intersections. Firstly, the trajectory of HVs is predicted in a sub-model, and the trajectories of the CAVs are further optimized in another sub-model [82]. These methods could minimize the delay. Ma et al. proposed 
an efficient dynamic programming and shooting heuristic (DP-SH) algorithm to optimize CAV trajectory and signal timing. At the beginning of the trajectory control section, the HV trajectory is predicted according to the entrance information of the upstream vehicle detector at the intersection [83]. The research studies listed above prove that the CAV cannot only improve self-experience but also influences the following vehicles and optimizes the overall traffic performance.

\subsection{Signal Controlling}

For the intersection signal control of $\mathrm{CAV}$, the control variables are the phase sequence and phase of signal timing. These variables should also be subject to appropriate constraints, such as maximum cycle length and maximum and minimum green time. Environment inputs are for arriving vehicles, which may comply with certain trajectory planning or speed recommendations. CAV-oriented intersection signal control is usually designed for a single intersection and then extended to a corridor and large area road network, and the objective function is modified to achieve the coordination of all intersections.

According to the control scheme, we can further divide the traffic-signal control for CAV into three types:

1. Actuated traffic-signal control enhanced by CAVs data.

2. Platoon-based traffic-signal control based on CAVs coordination.

3. Planning-based traffic-signal control based on CAVs coordination. The difference between these three types of control is the prediction of the future.

Actuated traffic-signal control enhanced by CAVs data simply estimates the current traffic status (such as queue length) and forecasts some relatively rough traffic indicators (such as average traffic) in the future. Then a control decision is made on this basis, such as extending or terminating the current phase. Platoon-based traffic-signal control based on CAVs coordination simplifies the problem of traffic-flow control to formation control, thus reducing the unknown. Planning-based traffic-signal control based on CAVs coordination has the most detailed information. It considers the detailed trajectory of each vehicle and optimizes the timing/phase of the signal within a certain time window by using a more accurate and complex model. At the same time, the robustness and flexibility of this method are relatively low [84].

Inductive signal control can dynamically adjust the timing parameters according to the arrival change of the real-time traffic volume. Because many existing research studies have considered the traffic-flow prediction based on the CAV data, this control method is also called adaptive traffic-signal control. This kind of control method can utilize the capacity of the intersection more effectively than the fixed-time signal control. The traditional trafficsignal control system uses inductive loop detectors to collect traffic information. These detectors are usually installed tens of meters upstream of the parking line; the information obtained is often inaccurate and spatially sliced, so it is not possible to present a complete change in traffic demand and arrival times of vehicles. The CAV provides a remedy for such problems. Depending on the exact position of the arriving vehicle, we can either extend or shorten the current phase, or add an extra phase to optimize the current signal control scheme [21].

Driven traffic-control depends more on real-time traffic information at the time and does not require high-traffic conditions in the future. In contrast, traffic prediction for traffic signal control based on formation is also based on planning, but the prediction accuracy of traffic flow is higher. Formation-based signal control identifies a platoon (or classifies a single vehicle as a pseudo-formation). These enable the vehicles in the queue to cross the intersection without serious disruption to their trajectory, thus improving overall traffic efficiency. Although the idea of traffic signal control based on formation has been proposed for several decades, it became a reality after the introduction of V2X technology. This is because V2X makes it possible to correctly identify vehicle formations and, thus, generate optimal signal-timing plans based on formation [85,86]. 
The method of signal optimization based on formation classifies the vehicles in the approach lane to each formation, ignoring the dynamics of the vehicles inside the formation and the mutual interference between the vehicles. In contrast, a planning-based approach that treats all vehicles at the same level can better describe real traffic conditions. In addition, formation-based signal optimization methods usually directly assume the known arrival distribution (such as Poisson distribution or uniform distribution), or estimate the arrival time of each formation, and assume that vehicles in each formation arrive uniformly. Planning-based methods usually estimate the actual arrival time of each vehicle and predict the traffic conditions in the advancing time range. Planning-based control has been widely studied by many researchers. The optimization model of planning-based control is usually an integer nonlinear optimization problem, especially when considering the trajectory of a single vehicle, which is difficult to solve. Some approximation and reprogramming methods are usually used to solve this problem. Dynamic programming is one of the most used techniques for redesigning and solving such control problems.

\subsection{Signal Timing-Vehicle Trajectory Collaborative Optimization}

In the study of traditional intersection control, signal timing and vehicle control are often studied separately. Characteristics of a single vehicle are rarely considered in the traditional traffic signal control system. However, signal control and vehicle control are interdependent. Signal timing affects the movement of individual vehicles, thus affecting vehicle performance (such as emissions and fuel consumption). At the same time, the performance of each vehicle is the key input of the traffic control method, that is, how to best adjust the signal timing.

However, information exchange between vehicles and traffic lights is rather limited in an off-network autopilot environment. The traffic light detects the arrival of a vehicle (usually a summary of the number of vehicles in a window of time) and adjusts its timing accordingly (usually passively). When approaching a junction, the driver sees the signal timing and adjusts his or her driving accordingly. Thus, the coupling control between signal and vehicle cannot be realized. With connected automated vehicles, information between traffic lights and individual vehicles can be exchanged in real time. We should make full use of this information to improve the traffic-control performance to achieve signal timing-vehicle trajectory co-optimization $[87,88]$.

In this type of study, the variables of the optimization problem are queue length, travel time, vehicle status (such as throttle and exhaust system status), etc. These variables should follow appropriate state equations, such as traffic-flow models and vehicle dynamic equations. The control inputs are signal time and phase, and vehicle operation (e.g., turning angle, accelerator pedal, etc.). These variables should also be appropriately constrained with traffic lights (such as minimum/maximum green time, cycle length, etc.) and traffic flows (such as car-following models). In general, the model of signal timing-vehicle trajectory collaborative optimization is complex, involving linear states, nonlinear states, discrete control inputs, continuous control inputs, and other complex constraints, which greatly increase the difficulty of solving this problem.

The existing research has proved that signal timing-vehicle trajectory collaborative optimization has great potential in improving the traffic control performance of intersections. In addition, when there are no off-network automated vehicles, signal timing-vehicle trajectory co-optimization may be easier to switch to non-signal control, thus further improving the performance of traffic control. At present, many problems with this method remain unsolved. Examples include how to combine signal control and vehicle control effectively, how to extend the signal timing-vehicle trajectory co-optimization method to control traffic corridor and network, and how to apply the method to real-world implementations [89,90].

In summary, this research will improve the efficiency of road-traffic information utilization, application level, and traffic co-control capability. It plays an important role in easing road-traffic congestion, improving road-traffic capacity, and improving road-traffic safety. It also provides new research directions and development opportunities for related 
disciplines and industries. It will promote the emergence and development of new ideas, new theories, new methods, new technologies, and new applications in interdisciplinary science $[91,92]$.

\section{Conclusion and Discussion}

According to the research status and development dynamics, it can be seen that many scholars and research institutions have conducted quite a lot of research on the implementation technologies, traffic state analysis, and experimental methods related to vehicle-road connectivity and cooperation. They have also conducted preliminary explorations on the traffic control methods for single intersections and special needs under the conditions of vehicle-road cooperation. However, they are still mainly focused on the physical system construction, adaptation analysis, and preliminary theoretical research and have not yet to develop the theory and experimental methods of mixed traffic flow cooperative control for intelligent connected vehicles. The vehicle terminal of the intelligent connected vehicles can respond to the signal control scheme, the surrounding vehicles, and the state of the road network, leading to significant changes in the operation mechanism of the hybrid traffic flow; at the same time, it can more abundantly and accurately collect the vehicle's position, speed, and other information under the conditions of the intelligent connected vehicles' hybrid traffic flow and can directly guide the intelligent connected vehicles. Thus, three control patterns can be formed, such as traffic signal control, intelligent connected vehicle guidance, and indirect control of conventional vehicles through intelligent connected vehicles, and its cooperative control mechanism and mechanism need to be studied urgently. With the popularization and use of intelligent connected vehicles, the study is proposed based on the urgent demand for cooperative control of urban road traffic flow and the significant changes in road traffic flow. It is a major directional, forward-looking, and trendy study. The related research can not only innovatively establish multi-modal vehicle traffic flow theory, but also provide critical support to countermeasure multi-modal vehicle traffic flow control problems and develop new-generation traffic-control systems.

The research on cooperative control of multi-intersections in the environment of intelligent connected vehicles is of great significance. First, under intelligent connected vehicle traffic-flow environment, traffic-control data input can be more abundant, detailed, and timely. Feedback optimization mechanism can be formed to reveal and establish the relationship between its traffic control and traffic status, as well as the operation and evolution of traffic flow between multi-intersections. It can not only greatly improve the accuracy of traffic control model and its algorithm itself, but also evaluate and feedback the traffic control effect by using the traffic flow operation condition obtained in real time. Second, under intelligent connected vehicle-traffic-flow environment, a new vehicle-road and vehicle-vehicle interaction can be provided. With making cooperation between vehicles each other and between driving environment and traffic signal control system, the controlling party (signal control system) and the controlled party (driving vehicle) can realize instant interaction, and the controlling party can obtain more detailed and accurate trafficcontrol information, including vehicle speed, vehicle position, vehicle path, etc., generating the optimal control scheme accordingly. At the same time, the controlled party can also obtain service information, such as speed guidance and active safety, making the execution of the control scheme more proactive and significantly improving the correlation and time-lag between traffic state detection and traffic control. Third, the organic relationship between the traffic states and the control targets and its parameters among the road-traffic system will be clearer, and the control methods will be more effective, forming active (feedforward) optimal control covering multiple strongly associated intersections. It can realize goal-oriented active traffic control driven by intelligent connected vehicle-traffic-flow data, especially for the complexity and randomness of the traffic system. Disseminating relevant information and taking proactive control measures on temporal and spatial scales before measurable inputs or disturbances have an adverse impact. 
In summary, due to the heterogeneous traffic flow consisting of human-driven vehicles, intelligent connected vehicles, and even self-driving vehicles lasting in a long time, the cooperative control technology of multi-intersections in the environment of intelligent connected vehicles is to establish the theory and method of cooperative control of multiintersections in the environment of intelligent connected vehicles through the analysis of their basic characteristics and cooperative mechanism, and to realize the cooperative control of new mixed-traffic flow for typical scenarios and application cases. It is of great significance to deepen the understanding and knowledge of the future new hybrid traffic flow, improve the control capability of the new hybrid traffic flow, and promote the development of intelligent connected vehicle cooperative control technology in the new hybrid traffic flow oriented to traffic efficiency.

Author Contributions: Conceptualization, L.Z. and Y.W.; formal analysis, L.Z. and H.Z.; investigation, L.Z.; writing — original draft preparation, L.Z. and Y.W.; writing—review and editing, Y.W. and Zhu Huaizhong. All authors have read and agreed to the published version of the manuscript.

Funding: This research was supported by National Key Research and Development Program of China (No. 2018YFB1600600) and National Natural Science Foundation of China (No. 61773293 and No. 52072264).

Institutional Review Board Statement: Not applicable.

Informed Consent Statement: Not applicable.

Data Availability Statement: Not applicable.

Acknowledgments: All authors are grateful for the resources provided by the Intelligent Transportation System Research Center of Tongji University.

Conflicts of Interest: The authors declare that there is no conflict of interest regarding the publication of this paper.

\section{References}

1. Panichpapiboon, S.; Pattara-Atikom, W. Evaluation of a neighbor-based vehicle density estimation scheme. In Proceedings of the 2008 8th International Conference on ITS Telecommunications, Phuket, Thailand, 14 October 2008; Volume 2008, pp. $294-298$.

2. Panichpapiboon, S.; Pattara-Atikom, W. Exploiting Wireless Communication in Vehicle Density Estimation. IEEE Trans. Veh. Technol. 2011, 60, 2742-2751. [CrossRef]

3. Sanguesa, J.A.; Fogue, M.; Garrido, P. Real-time Density Estimation in Urban Environments by Using Vehicular Communications, Wireless Days; IEEE: Piscataway, NJ, USA, 2012; pp. 1-6.

4. Barrachina, J.; Fogue, M.; Garrido, P.; Martinez, F.J.; Cano, J.-C.; Calafate, C.; Manzoni, P. Assessing vehicular density estimation using vehicle-to-infrastructure communications. In Proceedings of the 2013 IEEE 14th International Symposium on "A World of Wireless, Mobile and Multimedia Networks" (WoWMoM), Madrid, Spain, 4-7 June 2013; Volume 2013, pp. 1-3.

5. Barrachina, J.; Garrido, P.; Fogue, M.; Martinez, F.J.; Cano, J.-C.; Calafate, C.T.; Manzoni, P. A V2I-Based Real-Time Traffic Density Estimation System in Urban Scenarios. Wirel. Pers. Commun. 2015, 83, 259-280. [CrossRef]

6. Barrachina, J.; Sanguesa, J.A.; Fogue, M.; Garrido, P.; Martinez, F.J.; Cano, J.-C.; Calafate, C.T.; Manzoni, P. V2X-d: A vehicular density estimation system that combines V2V and V2I communications. In Proceedings of the 2013 IFIP Wireless Days (WD), Valencia, Spain, 13-15 November 2013; Volume 2013, pp. 1-6. [CrossRef]

7. Sanguesa, J.A.; Naranjo, F.; Torres-Sanz, V. On the Study of Vehicle Density in Intelligent Transportation Systems. Mob. Infor-Mation Syst. 2016, 2016, 1-13. [CrossRef]

8. Arbabi, M.H.; Weigle, M. Using Vehicular Networks to Collect Common Traffic Data; International Workshop on Vehicular Ad Hoc Networks: Beijing, China, 2009; pp. 117-118.

9. Arbabi, H.; Weigle, M.C. Monitoring free flow traffic using vehicular networks. In Proceedings of the Consumer Communications and NETWORKING Conference, Las Vegas, NV, USA, 9-12 January 2011; pp. 2720-2760.

10. Priemer, C.; Friedrich, B. A decentralized adaptive traffic signal control using V2I communication data, Intelligent Transportation Systems, 2009, ITSC '09. In Proceedings of the 12th International IEEE Conference, St. Louis, MO, USA, 4-7 October 2009.

11. Li, Z.; Wen, X.; Liu, Y.; Cheng, Y. Study on Methods of Traffic Estimation Under Connected and Autonomous Vehicles Mixed Traffic Flow; American Society of Civil Engineers: Reston, VA, USA, 2017.

12. Chen, X. Research on Road Traffic Information Collection and State Evaluation Method Based on CVIS; 2015; Available online: https: //www.researchgate.net/publication/347483085_Research_on_Traffic_Information_Monitoring_System_to_CVIS (accessed on 1 October 2021). 
13. Liu, X. Information Collection and Processing Method for Intelligent Traffic System based on IPv6; Jilin University: Changchun, China, 2011.

14. Nie, S. Simulation on the Interaction Relationship between Information Flow and Traffic Flow in the Inter-Vehicle Communi-Cation Scenario; Beijing Jiaotong University: Beijing, China, 2013.

15. Lee, W.H.; Hwang, K.P.; Wu, W.B. An intersection-to-intersection travel time estimation and route suggestion approach using vehicular ad-hoc network. Ad. Hoc. Netw. 2016, 43, 71-81. [CrossRef]

16. Zhou, H. Research on Traffic Information Collection and Processing Methods in Internet of Vehicles; Jilin University: Changchun, China, 2013.

17. May, A. Traffic Flow Fundamentals; Prentice Hall Inc.: Hoboken, NJ, USA, 1990; p. 1602190.

18. Gazis, D.C.; Herman, R.; Rothery, R.W. Nonlinear follow the leader models of traffic flow. Oper. Res. 1961, 9, 9132933. [CrossRef]

19. Olstam, J.J.; Tapani, A. Comparision of Car-following Models, Swedish National Road and Transport Research Institute (VTI); SE-581 95 Linkoping Sweden, Stockholm; Swedish National Road and Transport Research Institute: Linköping, Sweden, 2004.

20. Shunping, J.I.A.; Baohua, M.A.O. Research on CFCM: Car Following Model Using Cloud Model Theory. J. Transp. Syst. Eng. Inf. Technol. 2007, 7, 67-73.

21. Goodall, N.J.; Smith, B.L.; Park, B. Traffic Signal Control with Connected Vehicles. Transp. Res. Rec. J. Transp. Res. Board 2013, 2381, 65-72. [CrossRef]

22. Feng, Y.; Head, K.L.; Khoshmagham, S.; Zamanipour, M. A real-time adaptive signal control in a connected vehicle environment. Transp. Res. Part C Emerg. Technol. 2015, 55, 460-473. [CrossRef]

23. Gong, S.; Du, L. Cooperative platoon control for a mixed traffic flow including human drive vehicles and connected and au-tonomous vehicles. Transp. Res. Part B Methodol. 2018, 116, 25-61. [CrossRef]

24. Gipps, P.G. A model for changing decisions. Transp. Part B 1986, 20, 403-414. [CrossRef]

25. Mehmood, A. Integrated Simulation Model for Driver Behavior Using System Dynamic; University of Waterloo: Waterloo, ON, Canada, 2003.

26. Yang, Q.; Koutsopoulos, H.N. A microscopic traffic simulator for evaluation of dynamic traffic management systems. TransPortation Res. Part C 1996, 4, 113-129. [CrossRef]

27. Hidas, P. Modelling lane changing and merging in microscopic traffic simulation. Transp. Res. Part C Emerg. Technol. 2002, 10, 351-371. [CrossRef]

28. Sun, D.J.; Kondyli, A. Modeling Vehicle Interactions during Lane-Changing Behavior on Arterial Streets. Comput. Civ. Infrastruct. Eng. 2010, 25, 557-571. [CrossRef]

29. Talebpour, A.; Mahmassani, H.S.; Hamdar, S.H. Modeling lane-changing behavior in a connected environment: A game theory approach. Transp. Res. Part C Emerg. Technol. 2015, 59, 216-232. [CrossRef]

30. Nagel, K.; Schreckenberg, M. A cellular automaton model for freeway traffic. J. Phys. I 1992, 2, 2221-2229. [CrossRef]

31. Biham, A.O. Alan Middleton, Dov Levine. Self-Organization and a Dynamical Transition in Traffic Flow. Models Phys. Rev. A 1992, 46, R6124-R6127. [CrossRef] [PubMed]

32. Arai, K.; Sentinuwo, S.R. Effect of Driver Scope Awareness in the Lane Changing Maneuvers Using Cellular Automaton Model. Int. J. Adv. Res. Artif. Intell. 2013, 2, 6-12. [CrossRef]

33. Wang, J.; Gao, C.; Zhu, Z. Multilane Changing Model with Coupling Driving Intention and Inclination. Promet. Traffic Traffico 2017, 29, 185. [CrossRef]

34. Deng, J.; Feng, H. Multilane Cellular Automaton Model Based on the Lane-changing Mechanism. J. Transp. Syst. Eng. Inf. Technol. 2018, 18, 68-73.

35. Zhao, H.T.; Nie, C.; Li, J.R.; Wei, Y.-A. A two-lane cellular automaton traffic flow model with the influence of driver, vehicle and road. Int. J. Mod. Phys. C 2016, 27, 1650018. [CrossRef]

36. Li, S.; Yang, X. Competitive Lane-Changing Model Based on Cellular Automata. Transp. Stand. 2018, 4, 12-18.

37. Hidas, P. Modelling vehicle interactions in microscopic simulation of merging and weaving. Transp. Res. Part C Emerg. Technol. 2005, 13, 37-62. [CrossRef]

38. Kelsch, J.; Dziennus, M.; Köster, F. Cooperative Lane Change Assistant: Background, Implementation \& Evaluation. AAET 2015, 65-85. [CrossRef]

39. Heesen, M.; Baumann, M.; Kelsch, J. Investigation of Cooperative Driving Behaviour during Lane Change in a Multi-driver Simulation Environment. In Proceedings of the Human Factors and Ergonomics Society (HFES) Europe Chapter Conference Touluse, Toulouse, Frankreich, 10-12 October 2012; pp. 305-318.

40. Klein, L.A. Sensor Technologies and Data Requirements for ITS; TRID: The TRIS and ITRD Database: Washington DC, USA, 2001.

41. Cathey, F.; Dailey, D. Transit vehicles as traffic probe sensors. In Proceedings of the 2001 IEEE Intelligent Transportation Systems, Proceedings (Cat. No.01TH8585), Oakland, CA, USA, 25-29 August 2002; pp. 579-584.

42. Jazwinski, A.H. Stochastic Processes and Filtering Theory; Courier Dover Publications: Mineola, NY, USA, 2007.

43. Meier, J.; Wehlan, H. Section-wise modeling of traffic flow and its application in traffic state estimation. In Proceedings of the ITSC 2001, 2001 IEEE Intelligent Transportation Systems (Cat. No.01TH8585), Oakland, CA, USA, 25-29 August 2002; pp. 440-445.

44. Van Lint, J. Online learning solutions for freeway travel time prediction. IEEE Trans. Intell. Transp. Syst. 2008, 9, 38-47. [CrossRef]

45. Farradyne, P. Vehicle Infrastructure Integration (VII)-Architecture and Functional Requirements; Federal Highway Administration (FHWA): Washington, DC, USA, 2005. 
46. Wang, Q.; Hu, J.; Mu, C. A method of traffic flow measuring based on vehicle infrastructure integration system. In Proceedings of the 2009 IEEE Intelligent Vehicles Symposium, Xi'an, China, 3-5 June 2009; pp. 945-950.

47. Jenelius, E.; Koutsopoulos, H.N. Travel time estimation for urban road networks using low frequency probe vehicle data. Transp. Res. Part B Methodol. 2013, 53, 64-81. [CrossRef]

48. Argote, J.; Christofa, E.; Xuan, Y.; Skabardonis, A. Estimation of Arterial Measures of Effectiveness with Connected Vehicle Data. In Proceedings of the Transportation Research Board 91st Annual Meeting, Washington, DC, USA, 22-26 January 2012.

49. Goodall, N.J.; Smith, B.L.; Park, B.B. Microscopic estimation of freeway vehicle positions from the behavior of connected vehicles. J. Intell. Transp. Syst. 2016, 20, 45-54. [CrossRef]

50. Argote-Cabañero, J.; Christofa, E.; Skabardonis, A. Connected vehicle penetration rate for estimation of arterial measures of effectiveness. Transp. Res. Part C Emerg. Technol. 2015, 60, 298-312. [CrossRef]

51. Chen, Y.; Zhang, Y.; Hu, J.; Yao, D. Pattern Discovering of Regional Traffic Status with Self-Organizing Maps. In Proceedings of the 2006 IEEE Intelligent Transportation Systems Conference, Toronto, ON, USA, 17-20 September 2006; Volume 2006, pp. 647-652.

52. Ran, B.; Jin, P.J.; Boyce, D.; Qiu, T.Z.; Cheng, Y. Perspectives on Future Transportation Research: Impact of Intelligent Transportation System Technologies on Next-Generation Transportation Modeling. J. Intell. Transp. Syst. 2012, 16, 226-242. [CrossRef]

53. Fan, N.; Zhao, X.; Dai, M.; An, Y. Short-term traffic flow prediction model. J. Traffic Transp. Eng. 2012, 12, 114-119.

54. Wang, Y.; Wenjuan, E.; Tang, W.; Tian, D.; Lu, G.; Yu, G. Automated on-ramp merging control algorithm based on Internetconnected vehicles. IET Intell. Transp. Syst. 2013, 7, 371-379. [CrossRef]

55. Li, B.; Yao, D. Calculation of vehicle real-time position overcoming the GPS positioning latency with MEMS INS. In Proceedings of the IEEE International Conference on Service Operations and Logistics, and Informatics, Qingdao, China, 8-10 October 2014; pp. 248-254.

56. Chu, T.; Wang, J.; Codeca, L. Multi-Agent Deep Reinforcement Learning for Large-Scale Traffic Signal Control. IEEE Trans. Intell. Transp. Syst. 2019, 21, 1086-1095. [CrossRef]

57. Gong, Y.; Abdel-Aty, M.; Cai, Q.; Rahman, S. Decentralized network level adaptive signal control by multi-agent deep reinforcement learning. Transp. Res. Interdiscip. Perspect. 2019, 1, 100020. [CrossRef]

58. Liang, X.; Du, X.; Wang, G. Deep Reinforcement Learning for Traffic Light Control in Vehicular Networks. arXiv 2018, arXiv:1803.11115.

59. Jin, J.; Ma, X. A Multi-criteria Intelligent Control for Traffic Lights Using Reinforcement Learning. In Euro Working Group on Transportation European Conference on Operational Research; Springer: Cham, Switzerland, 2018.

60. Yu, C.; Ma, W.; Yang, X. A time-slot based signal scheme model for fixed-time control at isolated intersections. Transp. Res. Part B Methodol. 2020, 140, 176-192. [CrossRef]

61. Feng, S.; Feng, Y.; Yu, C.; Zhang, Y.; Liu, H.X. Testing Scenario Library Generation for Connected and Automated Vehicles, Part I: Methodology. IEEE Trans. Intell. Transp. Syst. 2021, 22, 1573-1582. [CrossRef]

62. Yu, C.; Feng, Y.; Liu, H.X.; Ma, W.; Yang, X. Corridor Level Cooperative Trajectory Optimization with Connected and Au-tomated Vehicles. Transp. Res. Part C Emerg. Technol. 2019, 105, 405-421. [CrossRef]

63. Yu, C.; Sun, W.; Liu, H.X.; Yang, X. Managing connected and automated vehicles at isolated intersections: From reservation- to optimization-based methods. Transp. Res. Part B Methodol. 2019, 122, 416-435. [CrossRef]

64. Yu, C.; Feng, Y.; Liu, H.X.; Ma, W.; Yang, X. Integrated optimization of traffic signals and vehicle trajectories at isolated urban intersections. Transp. Res. Part B Methodol. 2018, 112, 89-112. [CrossRef]

65. Feng, Y.; Yu, C.; Liu, H. Spatiotemporal intersection control in a connected and automated vehicle environment. Transp. Res. Part C Emerg. Technol. 2018, 89, 364-383. [CrossRef]

66. Yu, C.; Ma, W.; Han, K.; Yang, X. Optimization of vehicle and pedestrian signals at isolated intersections. Transp. Res. Part B Methodol. 2017, 98, 135-153. [CrossRef]

67. Feng, Y.; Yu, C.; Xu, S.; Liu, H.X.; Peng, H. An Augmented Reality Environment for Connected and Automated Vehicle Testing and Evaluation. In Proceedings of the 2018 IEEE Intelligent Vehicles Symposium (IV), Changshu, China, 26-30 June 2018; Volume 2018, pp. 1549-1554.

68. Leonard, K. Keeping the Promise of Connected Vehicle Technology: Toward an Era of Unprecedented Roadway Safety and Efficiency. TR News 2013, 285, 3-9.

69. Huang, L.-Y.; Yang, X.-G.; Du, R.-H. TJ-CTS: A Testbed of TongJi Cooperative Traffic System. In The First International Conference on Transportation Information and Safety; ASCE: Wuhan, China, 2011.

70. Wei, W.; Ma, W.; Yang, X. Simultaneous Optimization of Signal Timings, Departure Time and Dynamic Speed for Bus Priority Operations. In Proceedings of the Transportation Research Board 92nd Annual Meeting, Washington, DC, USA, 13-17 January 2013.

71. Osorio, C.; Bierlaire, M. A Simulation-Based Optimization Framework for Urban Transportation Problems. Oper. Res. 2013, 61, 1333-1345. [CrossRef]

72. Zhao, L.; Sun, J. Simulation Framework for Vehicle Platooning and Car-following Behaviors Under Connected-vehicle Environment. Procedia-Soc. Behav. Sci. 2013, 96, 914-924. [CrossRef]

73. Tideman, M.; Van Noort, M. A simulation tool suite for developing connected vehicle systems. In Proceedings of the 2013 IEEE Intelligent Vehicles Symposium (IV), Gold Coast, Australia, 23-26 June 2013. 
74. Kamalanathsharma, R.K.; Rakha, H.A.; Badillo, B. Simulation Testing of Connected Vehicle Applications in a Cloud-Based Traffic Simulation Environment. In Proceedings of the Transportation Research Board 93rd Annual Meeting, Washington, DC, USA, 12-16 January 2014.

75. Hafner, M.R.; Cunningham, D.; Caminiti, L. Cooperative Collision Avoidance at Intersections: Algorithms and Experiments. IEEE Trans. Intell. Transp. Syst. 2013, 14, 1162-1175. [CrossRef]

76. Lin, X.; Xu, J.; Wei, W.; Liang, Y.; Cai, L. Study on Construction of Connected-vehicle Network Simulation Experimental Platform and Its Parameters Correction. Logist. Scitech 2020, 43, 50-56.

77. Zhu, F.; Wen, D.; Chen, S. Computational Traffic Experiments Based on Artificial Transportation Systems: An Application of ACP Approach. IEEE Trans. Intell. Transp. Syst. 2012, 14, 189-198. [CrossRef]

78. Guler, S.I.; Menendez, M.; Meier, L. Using connected vehicle technology to improve the efficiency of intersections. Transp. Res. Part C Emerg. Technol. 2014, 46, 121-131. [CrossRef]

79. van den Broek, H.A.; Netten, B.D.; Hoedemaeker, M. The experimental setup of a large field operational test for cooperative driving vehicles at the A270. In Proceedings of the 13th International IEEE Conference on Intelligent Transportation Systems, Funchal, Portugal, 19-22 September 2010; pp. 198-203.

80. Woo, S.; Skabardonis, A. Flow-aware platoon formation of Connected Automated Vehicles in a mixed traffic with human-driven vehicles. Transp. Res. Part C Emerg. Technol. 2021, 133, 103442. [CrossRef]

81. Guo, Y.; Ma, J. DRL-TP3: A learning and control framework for signalized intersections with mixed connected automated traffic. Transp. Res. Part C Emerg. Technol. 2021, 132, 103416. [CrossRef]

82. Letter, C.; Elefteriadou, L. Efficient Control of Fully Automated Connected Vehicles at Freeway Merge Segments [J/OL]. Transp. Res. Part C Emerg. Technol. 2017, 80, 190-205. [CrossRef]

83. Ma, J.; Li, X.; Zhou, F.; Hu, J.; Park, B.B. Parsimonious shooting heuristic for trajectory design of connected automated traffic part II: Computational issues and optimization. Transp. Res. Part B Methodol. 2017, 95, 421-441. [CrossRef]

84. Guo, Q.; Li, L.; Ban, X. Urban traffic signal control with connected and automated vehicles: A survey. Transp. Res. Part C Emerg. Technol. 2019, 101, 313-334. [CrossRef]

85. Zhao, W.; Ngoduy, D.; Shepherd, S.; Liu, R.; Papageorgiou, M. A Platoon Based Cooperative Eco-Driving Model for Mixed Automated and Human-Driven Vehicles at a Signalized Intersection. Transp. Res. Part C 2018, 95, 802-821. [CrossRef]

86. He, Q.; Head, K.L.; Ding, J. PAMSCOD: Platoon-Based Arterial Multi-Modal Signal Control with Online Data. Transp. Res. Part C Emerg. Technol. 2012, 20, 164-184. [CrossRef]

87. Zheng, F.; Menendez, M.; Li, X.; Guler, I.; Van Zuylen, H. Modeling and managing mixed traffic with human-driven and automated vehicles. Transp. Res. Part C Emerg. Technol. 2020, 121, 102825. [CrossRef]

88. Kamal, M.A.; Hayakawa, T.; Imura, J.I. Development and Evaluation of an Adaptive Traffic Signal Control Scheme under a Mixed-Automated Traffic Scenario. IEEE Trans. Intell. Transp. Syst. 2020, 21, 590-602. [CrossRef]

89. Fang, S.; Yang, L.; Wang, T.; Jing, S. Trajectory Planning Method for Mixed Vehicles Considering Traffic Stability and Fuel Consumption at the Signalized Intersection. J. Adv. Transp. 2020, 2020, 1-10. [CrossRef]

90. Li, L.; Li, X. Parsimonious trajectory design of connected automated traffic. Transp. Res. Part B Methodol. 2019, 119, 1-21. [CrossRef]

91. Liu, B.; Shi, Q.; Song, Z.; Kamel, A.E. Trajectory planning for autonomous intersection management of connected vehicles. Simul. Model. Pract. Theory 2019, 90, 16-30. [CrossRef]

92. Zheng, F.; Liu, C.; Liu, X.; Jabari, S.E.; Lu, L. Analyzing the impact of automated vehicles on uncertainty and stability of the mixed traffic flow. Transp. Res. Part C Emerg. Technol. 2020, 112, 203-219. [CrossRef] 